\title{
Semantic oriented data structuration for MABS Application to BIM
}

\author{
Thomas Durif $^{1}$, Christophe Nicolle ${ }^{1}$, Nicolas Gaud ${ }^{2}$, and Stéphane Galland ${ }^{2}$ \\ 1 Le2I, Université de Bourgogne, Dijon, France \\ http://www.checksem.fr \\ \{thomas.durif, christophe.nicolle\}@checksem.fr \\ 2 IRTES-SET, UTBM, Belfort, France \\ http://set.utbm.fr \\ \{nicolas.gaud, stephane.galland\}@utbm.fr
}

\section{SHORT PAPER}

\begin{abstract}
This paper presents a multiagent-based simulation approach to qualify the usage of buildings from the design phase. Our approach combines ontology and evolution process based on machine learning algorithms. The ontology relies on semantic data structures for the representation of environment components, agent knowledge and all data generated during the simulation.
\end{abstract}

Keywords: ontology, modular ontology, Building Information Modeling, MultiAgent-Based Simulation

\section{Introduction}

This paper ${ }^{3}$ focuses on the application of multiagent-based simulation at the design phase of a civil engineering project. The output of the design phase is a 3D digital mockup that represents the future building. From this digital modeling, each stakeholder, according to their business, adds information to enrich the specification of the building model. All this data represents the Building Information Model (BIM) [2]. Each actor in this lifecycle uses specific norms and tools according to their domain of expertise.

To reduce the risk of heterogeneity the Building smart consortium developed a standard, called Industry Foundation Classes (IFC), that merges structural and geometrical data of a building to reduce heterogeneity. Unfortunatelly even if the structural, syntactic and semantic heterogeneity of data is limited, this standard doesn't deal with the gap between the conception of the building and its final use. To bridge this gap, our idea consists in simulating the behavior of the end users of a building to assess the quality of a building in terms of safety (e.g. security protocols), usability (e.g. the adaptation of capacity building are consistent with its

3 This work is partially funds by the Burgundy region 
use), comfort (e.g. limitation of movements required for routine tasks), etc. . To reach this goal, we use the field of Multiagent-based simulations (MABS), which are simulations that imply a group of interacting agents. Our approach combines a set of ontology (IFC ontology, multiagent ontology, environment ontology), with machine learning algorithms.

From there, we propose a new process for create MABS. It is based on an auto generated phase of different parts of MABS: environment, agents, interactions, simulations (specific features, logs, etc.) [5]. On the one hand, the semantically informed environment of the simulation is auto generated from an industrial file format of building representation IFC. On the other hand, minimal patterns of mobile learning agents are auto generated, based on specific features, from the agent part of the model we create. These elements will be discussed in the section entitled Approach, after introducing works in the field of semantics and MABS in the section entitled Related Works.

\section{Related work}

Various proposals includes semantics to improve Multi-Agent Based System (MABS). In [6], authors defined a taxonomy for an agent. Unfortunately, this task is complex because many approaches have different agents' definitions. It is possible to fit a large number of definitions, while limiting to a minimum specific constraints to certain areas. Semantics are the key point of this generic definition, as each expert can bring its own domain knowledge to each of the characteristics of a MABS. [3] focuses on the description of the interactions in an ontology. Goals are modeled as states of the environment. These goals are reached by means of inference based on the related ontology. Nevertheless, the contribution of semantics is limited to interactions including all MABS concepts. In [8], the work involves using an ontology to store the management of the agent's knowledge. Several specific features of ontologies are used (inferences, class hierarchy, queries, etc.). However, only the part affected to the agent's knowledge has been enhanced by the use of semantics. [4] describes a more complete approach. For managing the three basic elements of a multi-agent system : agents, environment and interactions. This approach represents the knowledge to lead a person to manipulate an object in a hazardous environment. It models the interactions of objects, but also the results produced by an interaction. This approach is also focused on training agents in a virtual environment. In this approach, the population process of the ontology is long. Each object required a manual specification for states, interactions, transitions, etc. All these approaches presented models don't consider the ontology as the central point for managing all data inherent to a simulation applied to MABS. Each approach addresses only a specific element of a MABS, that is either the agent, the simulation or the environment. To break these limits, our approach is based on a industrial process (building design) and its standards (IFC). Thus, the population of the ontology is dynamically made from the IFC files generated from CAD softwares. 


\section{Approach}

We propose a new methodology to create a MABS based on: automatic generation of the physical informed environment and self built smart agents. This feature provides the process of creating and managing a MABS. Our approach addresses four elements of a MABS : environment, agents, interactions and simulations. Our approach is based on a set of linked ontologies [10]. Each ontology is a domain ontology dedicated to the modeling of a specific area of expertise. In our context of knowledge managment, we use the term ontology to mean a specification of a conceptualization, as defined by [7]. This mixed of ontologies is depicted in figure 1.

\section{IFC Ontology $\bigcirc$ ○ Agent Ontology Interactive Environment Ontology}

Fig. 1. overview of the general ontology model

The IFC ontology contains the formal modeling of semantic and geometric information which model a building. These information are built during the design phase with CAD softwares. These softwares store the 3D digital mockup into an IFC files that are used to populate the IFC ontology. To populate this ontology we use a home made memory model in Java programming language. This first element allows our approach to be closed from industrial requirements. The 3D digital mockup is directly extract for CAD software not modeled using modeler software as usual in MABS proposals.

We propose a semantically aware environment automatically generated from the IFC standard [11]. The interactive environment ontology is dedicated to the modeling of the dynamical part of the simulation. this ontology uses some entities of the IFC ontology to combined building element (semantic and geometric definition) with smart objects (behavioral definition) [9]. A smart object is an object that can describe its own possible interactions. Thanks to the semantic obtain from the IFC ontology, the binding between smart objects and building objects is easier. A behaviour of a door can be connected with all IFCDoor instances defined in the IFC ontology. Thus the modeling time of the simulation is greatly reduced. The population process of this ontology is made by the domain expert, which defined the various states of a building objects and the different processes to move from an original state to a final state. The agent ontology describes the agent elements of the simulation. The agent are made of many concepts describing their ability to move, to interact with environmental objects, to interact with other agents, and to exchange requirements with the system managing the simulation. This definition of agent is derived from the ontology defined in [1] which aims to standardize the various definitions of multiagent-based simulation. The use of this ontology allow our approach to be compatible with different types of multi-agent based systems. The population of this ontology is made by the expert of the simulation. 
The top ontology integrates many entities of these various ontology. The simulation results, whatever their forms (3D modeling, knowledge, decisions, etc..) can be (re)used at any time during the simulation or other processing. This ontological foundation helps to provide a consistent basis for heterogeneous information, from both specific concepts related to contributing experts, and to the use of simulations and MABS.

A specific inference engine complete our architecture. It fixes the issues raised by agents that are unable to solve complex situations. The agents are autonomous and they need four basic characteristics: mouvement, perception, interaction and reasoning. To increase their knowledge, we force these agents to face simple situations. This results in an improved agents ability to face more complex behaviors. These agents will face simple situations to progress in knowledge that results in more complex behaviors. Secondly, we place these agents in real life simulation in environments based on real building plans.

In the followoing, we give a short overview of the process to build this simulation (see figure 2)

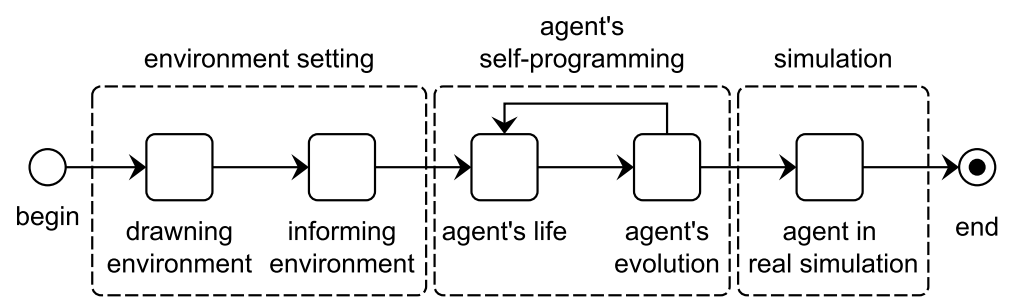

Fig. 2. overview of the process to build the simulation

To build a MABS, the environment and the agents need to be programmed. Firstly, the environment is created. IFC file and its implementation in the IFC ontology sets necessary information for structural and semantic perspectives. The environment needs an additional layer of information: everything related to the description of interactions. In the Interactive Environment Ontology, this information is already available. So as we already have all the information about the environment, this phase (environment setting) can be performed automatically.

Secondly, agents should be incorporated into the simulation. All the tools for defining agents are modeled in the Agent Ontology. From this ontology, the basic characteristics of agents are selected and extracted to create some basic agents. The designer of the simulation will put these agents in simple situations. A simple situation does not require from an agent specific knowledge to achieve the goal. Agents will accumulate knowledge about the behaviors needed on how to use objects. The designer sets a level for which it is considered that the agent has enough evolve in terms of knowledge to be confronted with the simulation in real conditions.

When this level is reached by the agents, they will be placed at different places of the environment with various profiles and they will move freely and test the usage of the building. Observation and treatment of agents' 
actions in certain contexts and according to their profiles will qualifying the usage of the building.

\section{Conclusion}

This paper outlined the steps necessary to build our ontology and the need to define semantic knowledge a MABS. Thanks to the ontology, our approach improve simulation process (reducing the definition of the environment, introducing a new way for agent progamming) and insert MABS in civil engineering projects. Future works concerns the development of the full platform combined with real building projects.

\section{References}

1. Béhé, F., Durif, T., Nicolle, C., Galland, S., Gaud, N., Koukam, A.: Ontology-based multiagent systems using Inductive Recommendations. In: 12th International Conference on Design \& Decision Support Systems in Architecture and Urban Planning. Eindhoven, PaysBas (Aug 2012)

2. Bernstein, P.G., China, F.: Building information modeling. Structural Engineer(Atlanta, Ga) 6(7), 18-21 (2005)

3. Chang, P.H.M., Chien, Y.H., Kao, E.C.C., Soo, V.W.: A knowledgebased scenario framework to support intelligent planning characters. In: Intelligent Virtual Agents. pp. 134-145. Springer (2005)

4. Edward, L., Amokrane, K., Lourdeaux, D., Barthes, J.P.: An ontology for managing a virtual environment for risk prevention. In: Integrated Intelligent Computing (ICIIC), 2010 First International Conference on. pp. 62-67. IEEE (2010)

5. Ferber, J.: Multi-agent systems: an introduction to distributed artificial intelligence, vol. 1. Addison-Wesley Reading (1999)

6. Franklin, S., Graesser, A.: Is it an agent, or just a program?: A taxonomy for autonomous agents. In: Proceedings of the Workshop on Intelligent Agents III, Agent Theories, Architectures, and Languages. pp. 21-35. ECAI '96, Springer-Verlag, London, UK, UK (1997)

7. Gruber, T.R.: Toward principles for the design of ontologies used for knowledge sharing. Int. J. Hum.-Comput. Stud. 43(5-6), 907-928 (Dec 1995)

8. Holmes, D., Stocking, R.: Augmenting agent knowledge bases with owl ontologies. In: Aerospace conference, 2009 IEEE. pp. 1-15. IEEE (2009)

9. Kallmann, M., Thalmann, D.: Modeling objects for interaction tasks. In: 9th Eurographics Workshop on Animation and Simulation. pp. 73-86 (1998)

10. Stuckenschmidt, H., Parent, C., Spaccapietra, S.: Modular Ontologies: Concepts, Theories and Techniques for Knowledge Modularization, vol. 5445. Springer (2009)

11. Vanlande, R., Nicolle, C., Cruz, C.: IFC and Buildings Lifecycle Management. Automation in Construction (18), 70-78 (Jan 2008) 